ORIGINAL ARTICLE

\title{
Short term effect of feedback on fitness and health measurements on self reported appraisal of the stage of change
}

\author{
K I Proper, A J van der Beek, V H Hildebrandt, J W R Twisk, W van Mechelen
}

Br J Sports Med 2003;37:529-534

See end of article for authors' affiliations

.....................

Correspondence to: Dr Proper, TNO Work and Employment, PO Box 718, NL- 2130 A'S Hoofddorp, The Netherlands;

K.Proper@arbeid.tno.nl

Accepted

1 November 2002
Background: An individual's current status of physical activity and nutrition and readiness to change can be determined using PACE assessment forms. Practitioners have suggested that feedback on the fitness and health components can produce a change in a subject's awareness of their behaviour and thereby lead to a beneficial change in stage of behaviour change.

Objective: To evaluate the short term effect of personalised feedback on fitness and health status on self reported appraisal of the stage of change.

Methods: A total of 299 civil servants were randomised to an intervention or a reference group. After having been tested for fitness and health, the intervention group received immediate feedback on their test results, whereas the reference group did not. PACE assessment forms were completed twice: before testing and after testing (reference group), or after testing and feedback (intervention group). The time interval between was one hour. The influence of feedback was determined using a $x^{2}$ test and analysis of variance. Results: On the basis of the $x^{2}$ test, no significant effect of feedback was found on the stage of change of physical activity, nor on the stage of change with regard to nutrition. Analysis of variance results showed no significant effect on the raw PACE score as to physical activity, intake of fruit and vegetables and dietary fat. However, a significant effect was observed on the PACE score of "calorie intake and weight management". Subjects in the intervention group significantly more often regressed on their PACE score on this topic than the reference subjects.

Conclusions: Feedback at baseline on measurements of an intervention study can influence PACE scores and can be considered as a small but relevant start of the intervention itself.
$\mathrm{T}$ he importance of regular physical activity is well established. ${ }^{1}$ Research has shown significant benefits of regular activity in the prevention of cardiovascular diseases and other chronic health problems. ${ }^{1-6}$ Despite these benefits, more than $60 \%$ of the adult population is not physically active at a sufficient level, and a quarter of adults in the United States are not active at all in their leisure time. ${ }^{178}$ Next to regular physical activity, the importance of healthy dietary habits has been emphasised in the prevention of many serious diseases such as cardiovascular diseases and some types of cancer. ${ }^{10}$ Still, most adults in Western countries do not meet nutrition guidelines. ${ }^{11-13}$ Furthermore, in both European countries and the United States, more than one third of the adults are overweight or obese. ${ }^{14-17}$ In addition, several studies have indicated that the prevalence of obesity has increased over the last few years. ${ }^{18} 19$ As the direct economic costs of treating obesity in the United States are estimated at over $\$ 70$ billion, ${ }^{20}$ increasing the proportion of adults who are regularly active and have a healthy diet is a great public health challenge. The above is supported by Joyner, ${ }^{21}$ who stated that, in developed countries, inadequate physical activity and obesity are two of the major health threats. The current epidemic of being overweight is caused largely by an environment that promotes excessive food intake and discourages physical activity. ${ }^{22}$ Weight gain is the result of a positive energy balance-that is, energy intake (through food intake) exceeds energy expenditure (through physical activity). Minor changes in food intake or physical activity could have large effects on body weight and the prevalence of obesity in the population. ${ }^{23}$ For interventions that promote physical activity and healthy dietary habits, adults are relatively accessible through the workplace. In the last few decades, many corporations have implemented health promotion programmes to improve the fitness and health of their employees. However, the effectiveness of such programmes is seriously hampered because target groups, in particular those with an unhealthy lifestyle who are not willing to change, are usually very hard to reach. In the last few years, new individual based approaches have been developed, focusing on the specific needs, motivations, and barriers of the individual. One of the models describing individual behaviour or the individual's readiness to change behaviour, is the transtheoretical model. This model suggests that individuals attempting to change their health behaviour move through a series of stages: ${ }^{24}(a)$ precontemplation (not intending to change behaviour); (b) contemplation (considering changing behaviour); (c) preparation (making small changes in behaviour); (d) action (actively engaging in behaviour change); (e) maintenance (sustaining the behaviour change over time). Integration of the transtheoretical model into health promotion interventions has appeared to be promising. ${ }^{25}$ One of the interventions based on the stages of change model is the patient centered assessment and counselling for exercise and nutrition (PACE) programme. ${ }^{26} 27$ Using the PACE assessment form, the individual's current status of physical activity and nutrition, and their readiness to change behaviour, can be determined. Hence, a counsellor can address specifically those processes that help the individual to progress to the next stage, given his/her specific situation. Professionals conducting fitness and health tests often have the impression that personalised feedback on test results already produces an awareness in the individual of their own behaviour. The hypothesis of this study is that personalised feedback on a person's fitness and 
health status leads to a change in their subjective appraisal of their stage of change, as measured with the PACE assessment form, which is supposed to be more in correspondence with reality. It is expected that employees who are not meeting the physical activity or dietary recommendations and who are not willing to become more physically active or adopt a more healthy diet (the precontemplators) may consider changing their behaviour to achieve a better PACE score when they become aware of poor test results. Thus the purpose of this study was to evaluate whether personalised feedback on fitness and health status, given immediately after completing a fitness and health test protocol, affects a person's subjective appraisal of their stage of behaviour change assessed by PACE scoring.

\section{METHODS \\ Study population}

A total of 600 employees within three municipal services of a Dutch town were invited to participate. Inclusion criteria were: (a) being a municipal civil servant; $(b)$ performing office work; (c) working at least 24 hours a week; (d) having a work contract lasting at least nine months after the measurements; (e) written informed consent. These criteria were set because the recruited study population participated in a trial investigating the effectiveness of a PACE-type intervention lasting nine months. Results of that intervention will be reported in a separate paper. Participation was voluntary. Units $(n=70)$, and thereby employees $(n=312)$, were randomised to an intervention group or a reference group. A total of 299 subjects who met the criteria for inclusion were measured. Figure 1 presents a flow chart including the number of participants. The study protocol was approved by the medical ethics committee of the VU University Medical Center.

\section{Randomisation}

The allocation schedule was made by a statistician, who was not involved in the project. Because of block randomisation, the allocation schedule was drawn up before the baseline measurements. However, subjects were informed of the

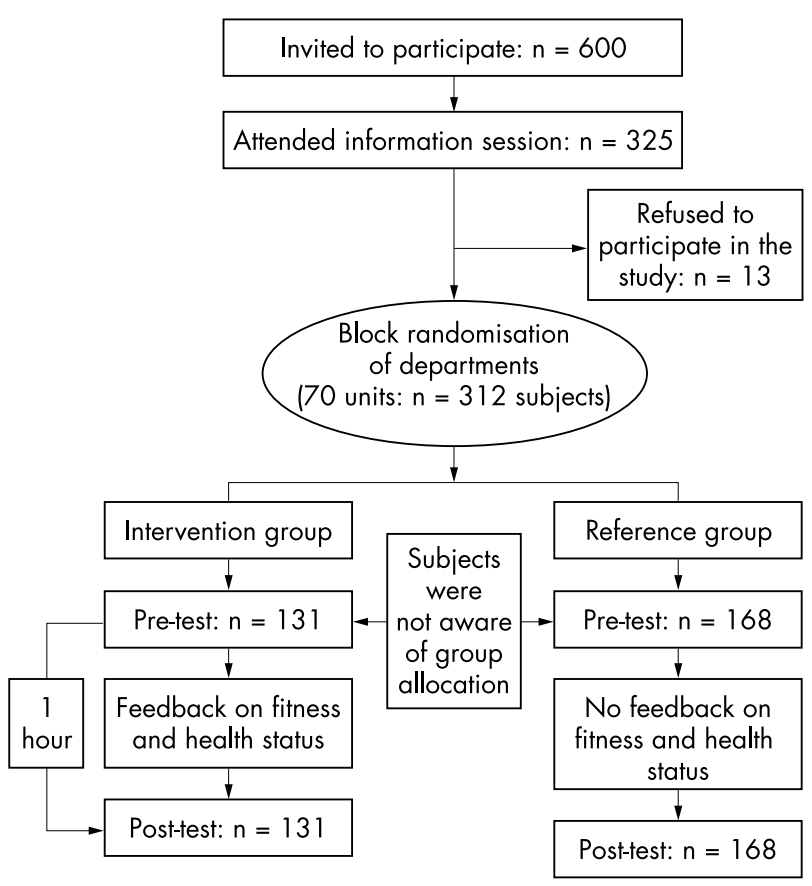

Figure 1 Flow chart of subjects in the trial. assignment after the baseline measurements. The assignment was carried out by the coordinator of the fitness and health tests, who did not perform any of the measurements. People who assisted with the tests were blinded to the allocation.

\section{Pace assessment forms}

PACE assessment forms were completed twice: $(a)$ before the fitness and health tests, and $(b)$ after the tests (reference group) or after the feedback given after the tests (intervention group). Subjects were not given the reason for completing the PACE forms twice. The time interval between the completion of the two PACE assessment forms was one hour. Each subject had to choose one out of eight statements that best fitted his/her current status of physical activity. According to the PACE counselling protocols, PACE assessment scores were classified into three stages of behaviour change. PACE score 1 referred to the stage of precontemplation, PACE scores 2-4 to the stage of contemplation/ preparation, and PACE scores 5-8 to the stage of action/ maintenance. With respect to nutrition, each subject had to tick one out of four statements on three topics: $(a)$ intake of fruit and vegetables; $(b)$ dietary fat intake; $(c)$ calorie intake and weight management. PACE score 1 referred to precontemplation, PACE scores 2-3 to contemplation/preparation, and PACE score 4 to action/maintenance. All four PACE assessment forms have shown good short term reproducibility (weighted $\kappa$ varied from 0.73 to 0.85 ; intraclass correlation coefficient varied from 0.76 to 0.89 ).

\section{Fitness and health tests}

The following variables were measured in all subjects: body weight, body height, waist and hip circumference, thickness of four skinfolds (biceps, triceps, suprailiac, and subscapular), resting heart rate, diastolic and systolic blood pressure (Nais blood pressure watch, type Fuzzy-logic, Tokyo, Japan), and total blood cholesterol (Reflotron; BoehringerMannheim, Mannheim, Germany). Moreover, subjects who were not using $\beta$ blockers performed a submaximal exercise test on a cycle ergometer (Lifecycle 9500 HR; Life Fitness, Chicago, Illinois, USA) according to the Åstrand protocol, by means of which the aerobic capacity $\left(\mathrm{VO}_{2} \mathrm{MAX}\right)$ was estimated. ${ }^{28}$ Subjects cycled for six minutes, except in the case of a difference between the fifth and sixth heart rate frequency larger than five beats, when subjects continued cycling for another minute. Waist and hip circumference, blood pressure, and skinfold thickness were measured twice and the means calculated. After these measurements, intervention subjects received some additional measurements as part of the PACE-type intervention in which they were invited to participate for nine months. These additional measurements included: shoulder flexibility (stick with a scale division in centimetres), hand grip strength (grip strength dynamometer; Takei Scientific Instruments Co, Tokyo, Japan), leg force (jump test using a springboard), hamstring flexibility (sit and reach test), heart function (electrocardiogram; Cardioperfect $4.1 \mathrm{st}$, The Netherlands), and lung function (peakflowmeter; Mini Wright, Clement Clarke). Body mass index was calculated as body weight $(\mathrm{kg})$ divided by body height squared $\left(\mathrm{m}^{2}\right)$. The percentage of body fat was calculated using the sum of the four skinfold thicknesses. ${ }^{29}$

\section{FEEDBACK}

Directly after measurements and testing, the intervention group consulted a sports doctor, who gave feedback in a standardised way on most of the test results, including waist circumference, percentage of body fat, diastolic and systolic blood pressure, total blood cholesterol, $\mathrm{VO}_{2} \mathrm{MAX}$, shoulder flexibility, hand grip strength, leg force, hamstring flexibility, and lung function. Test results on each of these variables 
were classified into two or more (not exceeding five) categories which served as criteria on which the feedback was based. Thus feedback was given in a standardised way using terms such as excellent, good, average, moderate, or bad, dependent on the number of categories. With respect to waist circumference and percentage of body fat, the criteria differed for men and women. For $\mathrm{VO}_{2} \mathrm{MAX}$, the criteria were dependent on both sex and age.

After standardised feedback, the sports doctor emphasised the role of regular physical activity and healthy dietary habits. Especially in the case of poor test results, the sports doctor encouraged subjects to improve their physical activity and dietary habits. Subjects in the reference group received neither their test results nor feedback on these results. In the case of extreme blood pressure values (diastolic $\geqslant 105 \mathrm{~mm} \mathrm{Hg}$ or systolic $\geqslant 180 \mathrm{~mm} \mathrm{Hg}$ ) and/or total blood cholesterol levels $(\geqslant 8.1 \mathrm{mmol} / \mathrm{l})$, a referral to a general practitioner was arranged. This applied for both the intervention and reference group.

\section{ANALYSIS}

Four groups indicating the pattern of change of behaviour stage were distinguished. These groups were based on the classification used by Marcus et $a^{30}$ and included: (a) stable sedentary (subjects who were in (pre)contemplation/preparation before test and after the test); $(b)$ stable active (subjects who were in action/maintenance at before and after the test); (c) adopters (subjects who moved in a progressive pattern from (pre)contemplation/preparation to action); $(d)$ relapsers (subjects who moved in a regressive pattern from action/maintenance back to (pre)contemplation/preparation). Subsequently, $\mathrm{a}^{2}$ test was conducted to examine the relation between the pattern of change in behaviour stage and the study group. In addition, of the raw PACE assessment scores, delta scores (post-test minus pre-test) were calculated, after which an analysis of variance was performed using the delta score as the dependent variable, and the study group and sex as the independent variables. $\alpha$ was set at 0.05 . As people in the action/maintenance stage do not need to change behaviour and were, for that reason, not the target group of the intervention, an additional subgroup analysis (analysis of variance) was conducted for the precontemplators and the contemplators/preparators only.

\section{RESULTS}

\section{Study population}

With the exception of sex, there were no significant differences in basic details, test results, and stage of change between the two study groups (tables 1 and 2). With respect to the current status of both physical activity and nutrition, only a minority of the subjects rated themselves as precontemplators. Three subjects, two of whom were in the reference group, received a referral to a general practitioner because of extreme values for blood pressure or blood cholesterol.

\section{Influence of feedback}

Most of the subjects in the intervention and reference groups did not change with regard to stage of physical activity and nutrition behaviour (table 3 ). Only a minority in both groups reported adopting a more physically active lifestyle or a healthier diet, or relapsing with regard to either. However, for calorie intake and weight management, a relatively large percentage of the intervention subjects $(12 \%)$ reported a relapse. The $\mathrm{x}^{2}$ test showed no significant differences between the study groups at the level of stage of behaviour change, nor did a stratified analysis for men and women. The results of the analysis of variance, however, showed a significant difference in raw PACE score for calorie intake and weight management ( $\mathrm{p}=0.002$; table 4$)$. The subgroup analysis, from which subjects in the action/maintenance stage were excluded, also showed a significant effect on calorie intake and weight management. Further, a separate analysis for men and women showed that the effect was mainly due to the men in our sample. It was shown that male intervention subjects significantly more often regressed on their PACE calorie intake and weight management score compared with male reference subjects, whereas no effect was seen among women. After having received feedback, $23 \%$ of the total intervention group, regressed on their PACE score on this topic. Of these, about half (53\%) moved from action/maintenance to contemplation/preparation. In the reference group, only $7 \%$ regressed on their PACE score with regard to this topic.

\section{DISCUSSION}

The purpose of this study was to evaluate whether instantaneous personalised feedback on fitness and health affects the self reported appraisal of the stage of behaviour change assessed by PACE forms. It was hypothesised that, after having been confronted with objective fitness and health status scores, a subject might change their subjective appraisal of their actual fitness and health profile. If so, they might change their self reported behaviour stage. Our findings showed no significant effect at the level of behaviour stage for either physical activity or nutrition. However, results of an analysis of variance showed that, after consultation, significantly more subjects in the intervention group regressed on their raw PACE score with respect to calorie

Table 1 Baseline characteristics of the study population

\begin{tabular}{lllll}
\hline & $\begin{array}{l}\text { Whole group } \\
(\mathbf{n = 2 9 9 )}\end{array}$ & $\begin{array}{l}\text { Intervention group } \\
(\mathbf{n}=131)\end{array}$ & $\begin{array}{l}\text { Reference group } \\
(\mathbf{n = 1 6 8 )}\end{array}$ & p Value \\
\hline Sex (\% women) & 34.1 & 27.5 & 39.3 & 0.03 \\
Age (years) & $44.1(8.7)$ & $44.6(7.9)$ & $43.6(9.3)$ & 0.33 \\
Body mass index (kg/m $\left.{ }^{2}\right)$ & $25.8(3.8)$ & $25.9(3.7)$ & $25.7(3.8)$ & 0.66 \\
Body fat (\%) & $28.9(6.5)$ & $28.7(6.6)$ & $29.1(6.5)$ & 0.58 \\
Waist circumference (cm) & $87.2(12.3)$ & $88.2(11.1)$ & $86.4(13.2)$ & 0.22 \\
Hip circumference (cm) & $99.7(6.9)$ & $99.3(7.1)$ & $100.3(6.6)$ & 0.23 \\
Diastolic blood pressure & $87.1(8.8)$ & $86.9(8.5)$ & $87.2(9.0)$ & 0.77 \\
(mm Hg) & & & & \\
Systolic blood pressure & $139.8(15.2)$ & $139.7(14.8)$ & $140.0(15.6)$ & 0.86 \\
(mm Hg) & & & & \\
Total cholesterol (mmol/l) & $5.5(1.0)$ & $5.5(1.0)$ & $5.4(1.0)$ & 0.24 \\
Estimated Vo ${ }_{2}$ MAX (litres/min) $3.1(0.8)$ & $3.1(0.8)$ & $3.0(0.8)$ & 0.17 \\
Resting heart rate (beats/min) $71.3(12.4)$ & $71.8(11.4)$ & $70.7(13.7)$ & 0.47 \\
\hline
\end{tabular}

Values are mean (SD). The $\mathrm{p}$ value refers to the $t$ test (for continuous data) or $\mathrm{x}^{2}$ test (for categorical data) to test for differences between the two study groups. 
Table 2 Baseline distribution of stages of change by treatment group

\begin{tabular}{|c|c|c|c|c|}
\hline \multirow[b]{2}{*}{ Stage } & \multicolumn{2}{|c|}{ Intervention group } & \multicolumn{2}{|c|}{ Reference group } \\
\hline & $\mathbf{n}$ & $\%$ & $\mathbf{n}$ & $\%$ \\
\hline \multicolumn{5}{|l|}{ Physical activity } \\
\hline Precontemplation & 0 & 0.0 & 4 & 2.4 \\
\hline Contemplation/preparation & 60 & 45.8 & 70 & 41.7 \\
\hline \multicolumn{4}{|l|}{ Fruit and vegetable intake } & 56.0 \\
\hline Precontemplation & 7 & 5.3 & 9 & 5.4 \\
\hline Contemplation/preparation & 67 & 51.1 & 84 & 50.0 \\
\hline Action/maintenance & 57 & 43.5 & 75 & 44.6 \\
\hline \multicolumn{5}{|l|}{ Dietary fat intake } \\
\hline Precontemplation & 24 & 18.3 & 32 & 19.0 \\
\hline Contemplation/preparation & 89 & 67.9 & 110 & 65.5 \\
\hline Action/maintenance & 18 & 13.7 & 26 & 15.5 \\
\hline \multicolumn{5}{|c|}{ Calorie intake and weight management } \\
\hline Precontemplation & 2 & 1.5 & 7 & 4.2 \\
\hline Contemplation/preparation & 77 & 58.8 & 101 & 60.1 \\
\hline Action/maintenance & 52 & 39.7 & 60 & 35.7 \\
\hline
\end{tabular}

intake and weight management, compared with the reference group. These findings suggest that personalised feedback does not immediately induce a change at the level of behaviour stage, but does induce a change at a more sophisticated level-that is, the raw PACE score.

The PACE assessment forms have shown to be of good short term test-retest reliability (weighted $\kappa, 0.73-0.85$; intraclass correlation coefficient, 0.76-0.89). Thus the effect shown on calorie intake and weight management was not due to the use of instruments that are not reliable.

A conceivable explanation for the effect of feedback on calorie intake and weight management is associated with the idea that a person has about his/her own body composition, in combination with the feedback given. In this study, feedback mainly concerned the results of the fitness and health tests. It could be that subjects had an initial self image about their body composition that did not correspond to the actual values. If so, the objective information received by the intervention subjects may adjust this self image towards the real body composition. In its turn, this adjustment may result in a change in PACE score, indicating a more realistic appraisal of the respondent's stage. In practice, this may be an important mechanism, because subjects who consider themselves as being in the action/maintenance stage, probably think they do not need to change their behaviour, whereas those rating themselves as contemplators are willing to improve their physical activity or dietary habits. Thus feedback can induce a more appropriate staging of the respondent.

It is intriguing why we did not find an effect on the other topics assessed by PACE. With respect to physical activity,

Table 3 Treatment condition and stage movement by stage of change at baseline

\begin{tabular}{|c|c|c|c|c|c|c|}
\hline \multirow[b]{2}{*}{ Baseline stage } & \multicolumn{2}{|l|}{ Progressed } & \multicolumn{2}{|l|}{ Stable } & \multicolumn{2}{|l|}{ Regressed } \\
\hline & Intervention & Reference & Intervention & Reference & Intervention & Reference \\
\hline \multicolumn{7}{|l|}{ Physical activity } \\
\hline Precontemplation & $x$ & $\begin{array}{l}50.0 \% \\
(n=2)\end{array}$ & $x$ & $\begin{array}{l}50.0 \% \\
(n=2)\end{array}$ & $x$ & $x$ \\
\hline Contemplation/preparation & $\begin{array}{l}15.0 \% \\
(\mathrm{n}=9)\end{array}$ & $\begin{array}{l}7.1 \% \\
(n=5)\end{array}$ & $\begin{array}{l}85.0 \% \\
(n=51)\end{array}$ & $\begin{array}{l}92.9 \% \\
(n=65)\end{array}$ & $x$ & $x$ \\
\hline Action/maintenance & $x$ & $x$ & $\begin{array}{l}91.5 \% \\
(n=65)\end{array}$ & $\begin{array}{l}88.3 \% \\
(n=83)\end{array}$ & $\begin{array}{l}8.5 \% \\
(n=6)\end{array}$ & $\begin{array}{l}11.7 \% \\
(n=11)\end{array}$ \\
\hline \multicolumn{7}{|l|}{ Fruit and vegetable intake } \\
\hline Precontemplation & $\begin{array}{l}14.3 \% \\
(\mathrm{n}=1)\end{array}$ & $x$ & $\begin{array}{l}87.7 \% \\
(n=6)\end{array}$ & $\begin{array}{l}100.0 \% \\
(n=9)\end{array}$ & $x$ & $\mathrm{x}$ \\
\hline Contemplation/preparation & $\begin{array}{l}3.0 \\
(n=2)\end{array}$ & $\begin{array}{l}2.4 \% \\
(n=2)\end{array}$ & $\begin{array}{l}97.0 \% \\
(n=65)\end{array}$ & $\begin{array}{l}97.6 \% \\
(n=82)\end{array}$ & $x$ & $x$ \\
\hline Action/maintenance & $x$ & $\mathrm{x}$ & $\begin{array}{l}94.7 \% \\
(n=54)\end{array}$ & $\begin{array}{l}92.0 \% \\
(n=69)\end{array}$ & $\begin{array}{l}5.3 \% \\
(n=3)\end{array}$ & $\begin{array}{l}8.0 \% \\
(n=6)\end{array}$ \\
\hline \multicolumn{7}{|l|}{ Dietary fat intake } \\
\hline Precontemplation & $x$ & $x$ & $\begin{array}{l}100.0 \% \\
(n=24)\end{array}$ & $\begin{array}{l}100.0 \% \\
(n=32)\end{array}$ & $\mathrm{x}$ & $x$ \\
\hline Contemplation/preparation & $x$ & $\begin{array}{l}2.7 \% \\
(n=3)\end{array}$ & $\begin{array}{l}100.0 \% \\
(n=89)\end{array}$ & $\begin{array}{l}97.3 \% \\
n=107)\end{array}$ & $x$ & $x$ \\
\hline Action/maintenance & $x$ & $x$ & $\begin{array}{l}72.2 \% \\
(n=13)\end{array}$ & $\begin{array}{l}84.6 \% \\
(n=22)\end{array}$ & $\begin{array}{l}27.8 \% \\
(n=5)\end{array}$ & $\begin{array}{l}15.4 \% \\
(n=4)\end{array}$ \\
\hline \multicolumn{7}{|c|}{$\begin{array}{l}\text { Dietary fat calorie intake and weight } \\
\text { management }\end{array}$} \\
\hline Precontemplation & $x$ & $x$ & $\begin{array}{l}100.0 \% \\
(n=2)\end{array}$ & $\begin{array}{l}100.0 \% \\
(n=7)\end{array}$ & $x$ & $x$ \\
\hline Contemplation/preparation & $\begin{array}{l}2.6 \% \\
(n=2)\end{array}$ & $\begin{array}{l}2.0 \% \\
(n=2)\end{array}$ & $\begin{array}{l}97.4 \% \\
(n=75)\end{array}$ & $\begin{array}{l}98.0 \% \\
(n=99)\end{array}$ & $x$ & $x$ \\
\hline Action/maintenance & $x$ & $\dot{x}$ & $\begin{array}{l}69.2 \\
(n=36)\end{array}$ & $\begin{array}{l}88.3 \% \\
(n=53)\end{array}$ & $\begin{array}{l}30.8 \% \\
(n=16)\end{array}$ & $\begin{array}{l}11.7 \% \\
(n=7)\end{array}$ \\
\hline
\end{tabular}




\section{Take home message}

- Feedback on measurements of health and fitness can produce a change in self reported appraisal of actual behaviour as measured with the PACE assessment form. Such feedback can thus be considered a small, but relevant starting point of the intervention itself.

subjects received objective information about their aerobic capacity $\left(\mathrm{VO}_{2} \mathrm{MAX}\right)$. In general, we think that most people do not know their $\mathrm{VO}_{2} \mathrm{MAX}$ value, as a result of which a reference value is lacking, making it impossible to compare their subjective idea with the objective value. For intake of fruit and vegetables and dietary fat, no feedback was given specifically on the consumption of these food items. Thus, in contrast with body composition, the feedback on physical activity and intake of fruit and vegetables and fat could not be translated directly into the categories available on the PACE forms. Moreover, for both fruit and vegetable intake and dietary fat intake, studies have shown discrepancies between the objective and subjective consumption, indicating that most people are not aware of the fact that their behaviour is unhealthy. ${ }^{31-33}$ In practice, creating awareness of actual consumption of food must therefore be the first, essential step to changing behaviour. ${ }^{32}$

A few issues arise from our study. The first involves the study population. Although randomly allocated to an intervention or reference group, the sample consisted of a self selected population. Unfortunately, the recruitment of subjects in studies on the promotion of physical activity often leads to inclusion of a self selected group of subjects. In general, workplace physical activity programmes often recruit only $20-30 \%$ of the workforce, and these also tend to be more fit and more aware of health issues than non-participants. ${ }^{34}$ Our study reached 50\% (299/600) of the employees invited to participate, which is thus relatively large. Furthermore, it appeared that $61 \%$ of our study population did not reach the moderate intensity physical activity recommendation. ${ }^{35}$ Compared with $60 \%$ (winter) and $47 \%$ (summer) of the general Dutch population who do not reach this physical activity standard, ${ }^{36}$ we conclude that our sample was similarly dominated by inactive people. However, as the sample included only a small percentage of precontemplators, we were not able to test the hypothesis that precontemplators in particular may consider changing their stage of behaviour to the contemplator/preparator stage, after having been confronted with a potentially poor fitness and/or health status. Future research involving precontemplators in particular would thus be very interesting.

Secondly, for individual counselling based on a PACE assessment, it should be kept in mind that subjects may report better lifestyle habits than they actually have. For calorie intake and weight management, our findings support this type of "misclassification". Although doubt can be cast on the validity of PACE assessment forms, a more relevant question is whether it is possible for such an instrument to be valid when it relies on self reporting of a behaviour that may not be accurately recalled.

Finally, the time interval between the completion of the two PACE assessment forms was short, one hour. For this reason, we cannot exclude the possibility of a first measurement effect. As no detailed information was given about the aim of the completion of two PACE assessment forms, it may be that subjects merely repeat their baseline score. This would underestimate any effect of feedback. For our purpose, the one hour interval is suitable, as the main focus was to determine whether subjects would change their PACE score after having been confronted with the test results that are then directly related to their behaviour. As we expected the influence of the feedback to be largest directly after having been confronted with the test results, the one hour interval was essential. To change actual behaviour, a longer interval is needed. This was realised, so a PACE-type intervention lasting nine months (see Methods section) was initiated directly after the baseline measurements.

To conclude, this study seems to support the notion that feedback on measurements can in itself induce changes in PACE scores and a more realistic appraisal of participants of their stage of change. Practitioners should therefore pay proper attention to feedback on measurements and fully use the potential of such feedback as the start of an intervention.

\section{CONCLUSION}

This study shows that personalised feedback on fitness and health status did not affect the PACE score on physical activity, intake of fruit and vegetables, and intake of dietary fat, but it did affect the PACE score on calorie intake and weight management. Subjects who received feedback appeared to regress on their behavioural stage with regard to this aspect more often than subjects without this feedback. Giving feedback on measurements at baseline of an intervention study can thus influence PACE scores and can be considered as a small, but important start of the intervention itself.

\section{ACKNOWLEDGEMENTS}

We thank Active Living Papendal, the local city authority of Enschede, and the occupational health service linked to the local authority for their important contributions to the project. We also thank Zon $\mathrm{Mw}$ and NOC*NSF for their financial support. Body@Work, Research Center Physical Activity, Work and Health is part of the Institute for Research in Extramural Medicine and is a joint initiative of TNO Work and Employment, the VU Medical Center, and TNO Prevention and Health.

\section{Authors' affiliations}

K I Proper, A J van der Beek, V H Hildebrandt, W van Mechelen, Body@Work, Research Center Physical Activity, Work and Health, TNOVU, TNO Work and Employment, EMGO-Institute and Department of Social Medicine, VU University Medical Center, Amsterdam,

Table 4 Treatment condition and PACE delta scores (post-test minus pre-test)

\begin{tabular}{|c|c|c|c|c|c|}
\hline & $\begin{array}{l}\text { Intervention group } \\
(\mathrm{n}=131)\end{array}$ & $\begin{array}{l}\text { Reference group } \\
(n=168)\end{array}$ & $p$ Value & $\begin{array}{l}\text { Difference in means } \\
\text { (intervention-reference) }\end{array}$ & $95 \% \mathrm{Cl}$ \\
\hline $\begin{array}{l}\text { Physical activity } \\
\text { Fruit and vegetable intake } \\
\text { Dietary fat intake } \\
\text { Calorie intake and weight }\end{array}$ & $\begin{array}{l}0.00(1.07) \\
-0.04(0.47) \\
0.11(0.61) \\
-0.18(0.66)\end{array}$ & $\begin{array}{l}-0.07(1.18) \\
0.01(0.39) \\
0.06(0.50) \\
0.05(0.50)\end{array}$ & $\begin{array}{l}0.67 \\
0.32 \\
0.57 \\
<0.01\end{array}$ & $\begin{array}{l}0.07 \\
-0.05 \\
0.05 \\
-0.22\end{array}$ & $\begin{array}{l}-0.21 \text { to } 0.32 \\
-0.15 \text { to } 0.05 \\
-0.09 \text { to } 0.16 \\
-0.35 \text { to }-0.08\end{array}$ \\
\hline
\end{tabular}

Values are mean (SD). The $\mathrm{p}$ value refers to the analysis of variance with the delta score as the dependent and study group and sex as the independent variables. $95 \% \mathrm{Cl}, 95 \%$ confidence interval. 
The Netherlands

J W R Twisk, Department of Clinical Epidemiology and Biostatistics and EMGO-Institute, VU University Medical Center

\section{REFERENCES}

1 US Department of Health and Human Services. Physical activity and health: a report of the Surgeon General. Atlanta, GA: US Department of Health and Human Services, Centers for Disease Control and Prevention, National Center for Chronic Disease Prevention and Health Promotion, 1996.

2 Lee IM, Sesso HD, Paffenberger RS. Physical activity and coronary heart disease risk in men: does the duration of exercise episodes predict risk? Circulation 2000;102:981-96.

3 Hu FB, Stampfer MJ, Colditz GA, et al. Physical activity and risk of stroke in women. JAMA 2000;283:2961-7.

4 Rainwater DL, Mitchell BD, Comuzzie AG, et al. Associations among 5-year changes in weight, physical activity, and cardiovascular disease risk factors in Mexican Americans. Am J Epidemiol 2000;152:974-82.

5 Kampert JB, Blair SN, Barlow CE, et al. Physical activity, physical fitness, and all-cause and cancer mortality: a prospective study of men and women. Ann Epidemiol 1996;6:452-7.

6 Vuori I. Does physical activity enhance health? Patient Educ Couns 1998:33(suppl):95-103.

7 Centers for Disease Control and Prevention. State-specific prevalence of participation in physical activity: behavioral risk factor surveillance system. Morb Mortal Wkly Rep 1996;45:673-5.

8 Caspersen CJ, Merritt RK, Stephens T. International physical activity patterns: a methodological perspective. In: Dishman RK, ed. Advances in exercise adherence. Champaign, IL: Human Kinetics, 1994:73-110.

9 US Public Health Service. The Surgeon General's report on nutrition and health. Washington, DC: US Department of Health and Human Services, 1988;DHHS (PHS) Publication No. 88-50210.

10 Jeffrey RW, Wing RR. Long-term effects of interventions for weight loss using food provision and monetary incentives. J Consult Clin Psychol 1995;63:793-6.

11 US Department of Agriculture. Nutrition and your health: dietary guidelines for Americans. Washington, DC: US Department of Health and Human Services, 1995.

12 Kristal AT, Shattuck AL, Henry JJ. Patterns of dietary associated with selecting diets low in fat: reliability and validity of a behavioral approach to dietary assessment. J Am Diet Assoc 1990;2:214-21.

13 Lissner L, Levitsky DA, Strupp BJ, et al. Dietary fat and the regulation of energy intake in human subjects. J Am Diet Assoc 1987;46:886-92.

14 European Commission, Directorate-General for Employment, Industrial Relations and Social Affairs. A PAN-EU survey on consumer attitudes to physical activity, body-weight and health. Luxembourg: Office for Official Publications of the European Communities, 1999

15 Martinez JA, Kearney JM, Kafatos A, et al. Variables independently associated with self-reported obesity in the European Union. Public Health Nutr 1999;2:125-33.

16 Daniel Vaz de Almeida M, Graca P, Afonso C, et al. Physical activity levels and body weight in a nationally representative sample in the European Union. Public Health Nutr 1999;2:105-13.
17 US Department of Health and Human Services. Physical activity and good nutrition: essential elements for good health. At-a-glance. Atlanta, GA: US Department of Health and Human Services, Centers for Disease Control and Prevention, 1998.

18 Troiano RP, Flegal KM. Overweight children and adolescents: description, epidemiology, and demographics. Pediatrics 1998;101:497-504.

19 Mokdad AH, Serdula MK, Dietz WH, et al. The spread of the obesity epidemic in the United States, 1991-1998. JAMA 1999;282:1519-22.

20 Colditz GA. Economic costs of obesity and inactivity. Med Sci Sports Exerc 1999;31(suppl):663-7.

21 Joyner MJ. Public health: what does it mean and who can benefit? Exerc Sport Sci Rev 2001;3:93-4.

22 French SA, Story M, Jeffery RW. Environmental influences on eating and physical activity. Annu Rev Public Health $2001 ; 22: 309-35$.

23 Seidell JC. The current epidemic of obesity. In: Bouchard C, ed. Physical activity and obesity. Champaign, IL: Human Kinetics, 2000:21-30.

24 Prochaska JO, Marcus BJ. The transtheoretical model: the applications to exercise. In: Dishman RK, ed. Advances in exercise adherence. Champaign, IL: Human Kinetics, 1994:161-80.

25 Marcus BH, Selby V, Niaura RS, et al. Self-efficacy and the stages of exercise behavior change. Res Q Exerc Sport 1992;63:60-6.

26 Patrick K, Calfas KJ, Sallis JF, et al. Basic principles of physical activity counseling: Project PACE. In: Thomas RJ, ed. The heart and exercise: a practical guide for the clinician. New York: Igaku-Shoin 1996:33-50.

27 Patrick K, Sallis JF, Long BJ, et al. A new tool for encouraging actvity: Project PACE. Physician and Sportsmedicine 1994;22:45-55.

28 Åstrand PO. Work test with the bicycle ergometer. Varberg: Monark-Crescent $A B$.

29 Durnin JVGA, Womersley J. Body fat assessed from total body density and its estimation from skinfold thickness: measurements on 481 men and women aged from 16 to 72 years. Br J Nutr 1974;32:77-97.

30 Marcus BH, Simkin LR, Rossi JS, et al. Longitudinal shifts in employees' stages and processes of exercise behavior change. Am J Health Promot 1996; 10:195-200.

31 Brug J, Van Asssema P, Kok GJ, et al. Self-rated dietary fat intake: association with objective assessment of fat, psychosocial factors, and intention to change. $J$ Nutr Educ 1994;26:218-23.

32 Lechner L, Brug J, De Vries H. Misconception of fruit and vegetable consumption: differences between objective and subjective estimation of intake. J Nutr Educ 1997;29:313-20.

33 Bowen DJ, Fries E, Hopp HP. Effects of dietary feedback on behavioral and psychological variables. J Behav Med 1994;17:589-604.

34 Lovato CY, Green LW. Maintaining employee participation in workplace health promotion programs. Health Educ Q 1990;17:73-88.

35 Pate RR, Pratt M, Blair SN, et al. Physical activity and public health, a recommendation from the Centers for Disease Control and Prevention and the American College of Sports Medicine. JAMA 1995;273:402-7.

36 Hildebrandt VH, Urlings IJM, Proper KI, et al. [Are Dutch people physically active (enough)?] (In Dutch.) In: Hildebrandt VH, Ooijendijk WTM, Stiggelbout M, eds. Trendrapport bewegen en gezondheid 1998/1999. Lelystad: Koninklijke Vermande, 1999:23-9. 